\title{
The amyloidogenic peptide amyloid beta(16-22) displays facet dependent conformation on metal surfaces
}

\author{
Kieran P. Somers and David L. Cheung* \\ School of Chemistry, National University of Ireland Galway, Galway, H91 TK33, Ireland
}

\begin{abstract}
Currently, it is not understood how metal nanoparticles influence the formation of protein fibrils, although recent literature highlights that the shape and chemical composition of such nanoparticles can strongly influence the process. Understanding this process at a fundamental level can potentially unlock routes to the development of new therapeutics, as well as novel materials for other applications. This requires a microscopic picture of the behaviour of amyloidogenic proteins on metal surfaces. Using replica exchange molecular dynamics simulations we investigate the adsorption of the model amyloidogenic peptide, A $\beta(16-22)$, on different gold and silver surfaces. While the peptide adsorbs onto these different surfaces, the adsorption can be considered to be enthalphically driven for the gold surfaces and entropically driven for silver. The conformation of the peptide on gold surfaces also shows a strong facet dependence for gold, with fibril-like conformations being promoted in the 100 surface and inhibited on the 111 surface. A smaller degree of facet dependence is seen for silver with the peptide behaving similar on both of these. The difference in the facet dependence can be related to the difference between direct adsorption onto the gold 111 surface, with a preference towards indirect (water mediated) adsorption onto the other surfaces. This new information on the behaviour of an amyloidogenic peptide on metal surfaces can give insight into the size-dependent effect of nanoparticles on fibril formation and the use of surfaces to control fibrillation.
\end{abstract}




\section{INTRODUCTION}

Research on fibril formation via protein agglomeration into amyloid fibrils ${ }^{1,2}$ is of great importance in a wide variety of fields, ranging from microbiology to biochemistry to materials science and medicine. Whilst fibril formation is involved in many normal biological functions, the abnormal aggregation of proteins into amyloids and subsequently fibrils, or "plaques", has been implicated in the pathology of numerous human diseases, and specifically, in the onset of a group of diseases eponymously encompassed by the term "amyloidosis". These include degenerative diseases, such as Alzheimer's and Parkinson's disease, which due to increasing lifespans are becoming more prevalent ${ }^{3}$. As well as pathogenic fibrils, so-called functional fibrils have been identified; these play a role in many processes such as surface adhesion $^{4}$ and biofilm formation ${ }^{5}$. Outside the field of human pathology, many are trying to exploit protein fibrillation to develop novel materials ${ }^{6,7}$.

While protein fibrils can form under a range of conditions, the formation of protein fibrils is known to be influenced by surfaces and interfaces ${ }^{8,9}$, both natural and synthetic. In vivo, fibrils are often associated with the cell membrane ${ }^{10}$, which has been implicated in amyloiddiseases, such as Alzheimers ${ }^{11-14}$ and type-II diabetes ${ }^{15}$. The presence of synthetic surfaces and interfaces can also impact fibril formation ${ }^{16}$. This can be exploited in the creation of amyloid fibrils for materials applications ${ }^{6,17-19}$ and can also influence the interaction of manmade materials with synthetic systems ${ }^{20,21}$. Recently, particular interest has focused on the interaction of nanomaterials with proteins and the potential for nanoparticles to influence fibril formation ${ }^{22-24}$.

Noble metal nanoparticles have attracted particular attention. They been investigated for a number of biomedical applications, such as drug delivery or medical imaging, so understanding their interaction with proteins is of significant importance. The effect of gold nanoparticles and nanoclusters on the formation of amyloid fibrils has also been investigated $^{25}$. This work found a size dependent effect with nanoclusters and small gold nanoparticles inhibiting fibrillation while larger ones promote this ${ }^{24}$. Nanoparticles consisting of other noble metals, such as silver or platinum, have also been investigated for biomedical applications ${ }^{26-31}$. Their affect on fibrillation behaviour is less clear so it is of interest to determine the how different metal surfaces interact with amyloidogenic proteins ${ }^{22}$. As well as depending on the metal the interactions between proteins and surfaces can depend 
on the exposed crystal face, with facet dependent adsorption being seen for metal ${ }^{32,33}$ and oxide surfaces $^{34,35}$. Differential adsorption of proteins onto surfaces can be used to control the formation of nanoparticles ${ }^{36,37}$ and lead to the size dependent effect of nanoparticles on protein fibrillation ${ }^{38}$.

While the link between surface properties and fibrillation is of significant interest, our understanding of this is still developing. The effect of surfaces on the formation of amyloid fibrils depends on the interplay between protein and surface properties ${ }^{9}$. Commonly changes to the surface hydrophobicity have been investigated. For amyloid-beta fibrillation is enhanced on hydrophobic surfaces and inhibited in hydrophilic surfaces ${ }^{39}$, while the opposite behaviour is seen for amylin ${ }^{40-42}$. For both proteins, however, the concentration on the hydrophobic surfaces are higher than on the hydrophilic ones, suggesting that changes in fibrillation is not simply due to changes in protein concentration, rather the effect of surface induced conformational change and protein mobility on surfaces ${ }^{39}$ are important in fibril formation on surfaces ${ }^{9}$. A wide range of experimental methods have been used to investigate protein conformation and fibrillation on surfaces ${ }^{43,44}$ However, these have difficulty in resolving the single molecular structure that limits their ability to investigate the early stages of fibril formation.

As it is able to directly access the molecular level molecular dynamics simulation provides a route to investigate protein behaviour on surfaces ${ }^{45,46}$. In particular recent work has applied it to the investigation of a number of amyloidogenic proteins, including amyloid beta $^{47-49}$, amylin ${ }^{38,50}$, and beta-microglobulin ${ }^{51}$ on solid surfaces. These simulations have been enabled through advances in simulation methodology, such as replica exchange ${ }^{52}$ or metadynamics ${ }^{53}$ simulations, that allow for the sampling of the diverse conformational ensemble of these proteins and accurately describe the protein-surface interaction. Due to the importance of electrostatic effects metal surfaces have proven to be particularly challenging for force field based modelling ${ }^{54}$. Recent work has derived simulation models for noble metals including polarization, which can account for these. In particular the GolP ${ }^{55,56}$ and related models introduce polarization through the inclusion of rigid dipoles on the metal atoms provides a computationally efficient method for approximating electrostatic effects into modelling biomolecular-interface interactions. These models have been used to investigate the adsorption of proteins onto effect of gold and silver surfaces, including fibril forming proteins $^{38,47,48,51}$ and gold and silver binding peptides ${ }^{57,58}$. 
In this paper we use molecular dynamics simulations to investigate the behaviour of a model amyloidogenic peptide, the $\mathrm{A} \beta(16-22)$ fragment of amyloid-beta ${ }^{59}$, on metal surfaces. The main goal is to investigate how changes to the surface can affect conformation of $\mathrm{A} \beta(16-$ 22) and its fibrillation behaviour. Specifically the 111 and 100 gold and silver surfaces are investigated, which allows for comparison between different metals and facets. Note that while metal nanoparticles commonly have ligands molecules on their surfaces, which affects their interaction with biomolecules, depending on the ligand density interactions between the underlying metal and proteins are possible. This is particularly the case where ligands are non-uniformly distributed on the nanoparticle surface ${ }^{60}$. Interaction with metal surfaces are also likely in materials and biotechnology applications.

\section{METHODOLOGY}

All simulated systems contained a single A $\beta(16-22)$ peptide (KLVFFAE). The termini and polarizable residues ( $\mathrm{K}$ and $\mathrm{E}$ ) were charged, as appropriate for $\mathrm{pH}$ 7. For bulk simulations this was solvated in a water box with water molecules overlapping with the peptide removed, giving a total number of water molecules of 2117 .

To examine the effect of crystal facet on adsorption both 111 and 100 surfaces were investigated. The 111 crystal faces consisted of a $20 \times 12$ unit cells, while the 100 faces consisted of $20 \times 20$ unit cells. In both cases the surfaces consisted of 5 layers of atoms. Lattice parameters of $4.14 \AA$ and $4.16 \AA$ were used for $\mathrm{Au}$ and $\mathrm{Ag}$ surface respectively, consistent with previous work ${ }^{61,62}$. Positions of the surface atoms (except for the mobile charge sites) were held fixed in the simulations. Note that while surface reconstruction can change the structure of metal surfaces, previous work has shown that allowing the surface to change its structure has only a minor effect on the simulation results ${ }^{58}$. The peptide was placed approximately $10 \AA$ from the surface and the system was solvated, with water molecules overlapping with the peptide and surface removed. Each system contained approximately 7800 water molecules.

The peptide was modelled using the charmm $22^{*}$ force field ${ }^{63-65}$, with the modified charmm-TIP3P ${ }^{66}$ water model. This combination has been shown to give a good description of the $\mathrm{A} \beta(16-22)$ peptide $^{67}$ Gold and silver surfaces were modelled using the GolP-Charmm ${ }^{61,68}$ and AgP-Charmm ${ }^{62}$ models respectively. 
For all cases the initial structure was energy minimised, with a tolerance of $10^{3} \mathrm{~kJ} \mathrm{~mol}^{-1} \mathrm{~nm}^{-1}$. For the surface simulations the $z$-box length was adjusted so the water density far for the surface was equal to the bulk water density. Simulations in the NVT ensemble (for surfaces) and NPT ensemble (for bulk simulations) were then performed for $100 \mathrm{~ns}$ to equilibrate the systems.

In order to enhance sampling of protein conformations replica exchange with solute tempering (REST) was employed ${ }^{69,70}$. This is a variation on replica exchange molecular dynamics $^{71}$, where the temperature varies only for a subset of the system, in this case the protein. The temperature scaling was performed by scaling the protein-protein and proteinsolvent interactions by a factor depending on the effective temperature. Specifically the potential energy was given by ${ }^{70}$

$$
E_{i}=\beta_{i} E_{p p}+\beta_{i}^{1 / 2} E_{p s}+E_{s s}
$$

where $E_{p p}, E_{p s}$, and $E_{s s}$ are the protein-protein, protein-solvent, and solvent-solvent interaction and the scaling factor $\beta_{i}=T_{0} / T_{i}$. For all systems the effective temperature was in the range $300 \mathrm{~K}$ to $440 \mathrm{~K}$; six replicas with scaling factors (effective temperatures) 1 (300 K), $0.926(324 \mathrm{~K}), 0.858(350 \mathrm{~K}), 0.795$ (378 K), 0.736 (408 K), and 0.682 (440 K).

Surface simulations were performed in the NVT ensemble at a temperature of $300 \mathrm{~K}$, using the velocity-rescaling algorithm of Bussi et al ${ }^{22}$ with a relaxation time of 0.2 ps. For bulk solution simulations were performed in the NPT ensemble with a temperature of $300 \mathrm{~K}$ and pressure of 1 atmosphere. The pressure was controlled using the ParinelloRahman barostat ${ }^{73}$ with relaxation time of 2 ps. All simulations were performed with periodic boundary conditions in all dimensions. The Gromacs molecular dynamics package ${ }^{74}$ (version 4.6.7) was used for all the simulations, with a modified version of the PLUMED library ${ }^{75,76}$ used to implement REST.

Simulations were performed for 200 ns (surface) and 400 ns (solution). These simulation times were found to be sufficient for the cluster entropy

$$
S_{\text {cluster }}=\sum_{i} p_{i} \ln p_{i}
$$

to plateau. In Equation 2 the sum runs over the unique peptide conformations and $p_{i}$ is the probability of the $i$ th conformation. The unique conformations were determined using the method of Daura ${ }^{77}$, with two conformations being in the same cluster if the $\mathrm{C}_{\alpha} \mathrm{RMSD}$ is less than $1 \AA$. 
Analysis of the simulations was performed using a combination of standard gromacs utilities and custom-written python scripts using the MDAnalysis library ${ }^{78}$. Simulation snapshots were generated using VMD (visual molecular dynamics) ${ }^{79}$. Unless otherwise stated analysis was performed over the last $100 \mathrm{~ns}$ of each simulation. For the surface simulations analysis This was taken to be when the closest distance between the surface and a heavy (nonhydrogen) atom in the peptide is less than $7 \AA$. This cut-off was large enough to encompass both direct contacts between the peptide and surface and cases where adsorption is mediated by water molecules.

Average peptide-surface interaction energies $\left(E_{\text {int }}\right)$ were determined from

$$
\left\langle E_{\text {int }}\right\rangle=\left\langle E_{\text {peptide-surface }}\right\rangle-\left\langle E_{\text {peptide }}\right\rangle-\left\langle E_{\text {surface }}\right\rangle
$$

where $E_{\text {peptide-surface }}$ is the potential energy of the peptide-surface system and $E_{\text {peptide }}$ and $E_{\text {surface }}$ are the potential energies of the peptide and surface on their own. The averages were calculated across the simulation trajectories using the gromacs rerun option.

The conformational ensemble of the peptide in the different simulations was determined using a cluster analysis based on the $\mathrm{C}_{\alpha}$ RMSD, using the method of Daura et al with a cut off of $1 \AA$. To analyse the differences between the conformational ensemble on the metal surfaces and in bulk solution a combined cluster analysis was preformed. In this conformations from the last 100 ns of each simulation (for surface simulations this included only conformations where the peptide is in contact with the surface) were grouped together and a single cluster analysis was performed.

\section{RESULTS}

\section{A. Adsorption behaviour of $\mathbf{A} \beta(16-22)$ on gold and silver surfaces}

The adsorption of $\mathrm{A} \beta(16-22)$ onto different metal surface depends on both the metal and exposed facet. For the Au111 surface the peptide is strongly bound to the surface, with the separation between the peptide centre-of-mass and surface being between 5 and $10 \AA$ throughout the simulation (Figure 1(a)). This strong adsorption is consistent with previous simulations of $\mathrm{A} \beta(16-22)$ on Au111 surfaces $^{47}$. In contrast to the Au111 surface adsorption onto the other surfaces is weaker, with the peptide able to desorb from the surface (Figure 1(a)). The weaker adsorption can be seen by considering the $P(z)$ histogram 
of peptide-surface separations (Figure 1(b)). For the Au111 surface this is tightly peaked, with the peptide only found within $10 \AA$ of the surface, while the distribution for the other surfaces is broader. Notably for the silver surfaces the peak is further away from the surface, indicative of water mediated adsorption onto the surface, similar to that seen for single amino acids onto silver ${ }^{57}$.

Estimating the adsorption free energy from $F(z)=-R T \ln P(z)$ gives adsorption free energies of between -3 and $-4 \mathrm{~kJ} \mathrm{~mol}^{-1}$ for the Au100, Ag111, and Ag100 surfaces, consistent with the relatively weak binding to the surface. The limited range of separations sampled for the Au111 makes it impossible to estimate the adsorption free energy in a similar manner, although the strong adsorption is consistent with the adsorption free energies $\left(-62 \mathrm{~kJ} \mathrm{~mol}^{-1}\right)$ determined previously ${ }^{47}$, albeit using a different force field. The difference in adsorption strengths between the 111 and 100 facets is consistent with previous simulations of goldbinding peptides on different gold facets ${ }^{58}$.

The average peptide-surface interaction energy, $E_{i n t}$, was calculated for each of the surfaces (Table I). A significantly stronger interaction was found with the Au111 surface compared to the other surfaces.

\begin{tabular}{ll}
\hline Surface & $E_{\text {int }} / \mathrm{kJ} \mathrm{mol}^{-1}$ \\
\hline Au111 & $-188 \pm 28$ \\
Au100 & $-57 \pm 48$ \\
Ag111 & $-26 \pm 26$ \\
Ag100 & $-16 \pm 10$ \\
\hline
\end{tabular}

TABLE I. Peptide-surface interaction energies calculated from MD simulations. Uncertainties estimated from standard deviation.

To gain more insight into the behaviour of the peptide on the different surfaces the separation between the centre-of-mass of each residue and surface are shown in Figure 1(c). For the Au111 surface the two phenylalanine residues (F19 and F20) are consistently in contact with the surface. This is in line with previous simulations of $\mathrm{A} \beta(16-22)$ on the Au111 surface ${ }^{47}$ and with the strong adsorption of phenylalanine onto Au111 surfaces ${ }^{61}$. The importance of the phenylalanine residues on the adsorption can also be seen from the average separation between each residue and the surface. Both F19 and F20 have $\bar{z} \sim 3-4 \AA$ 
(a)

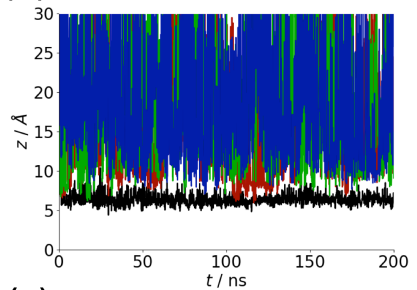

(c)
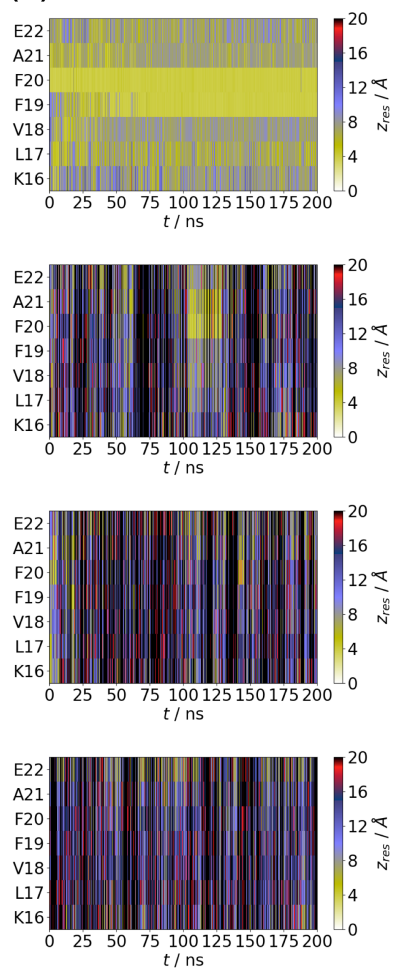

(b)
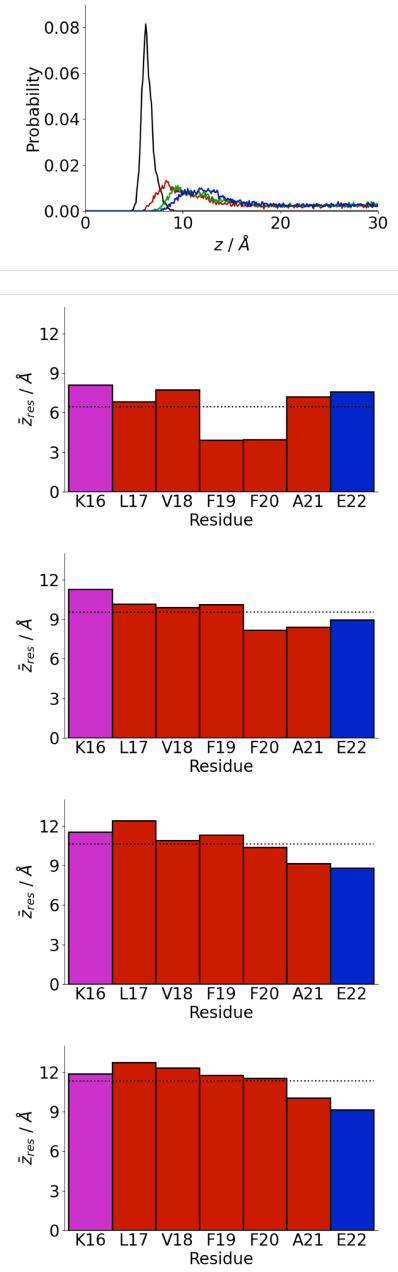

FIG. 1. (a) A $\beta(16-22)$ centre-of-mass position. Black, red, green, and blue lines denote Au111, Au100, Ag111, and Ag100 respectively. (b) Probability histograms (averaged over last 100 ns of simulations) for A $\beta(16-22$ ) on Au111 (black), Au100 (red), Ag111 (green), and Ag100 (blue). (c) Residue centre-of-mass-surface separations (left) and average residue centre-of-mass-surface separations (right). From top-to-bottom Au111, Au100, Ag111, and Ag100. Red, blue, and magenta denote hydrophobic, negatively-charged, and positively-charged residues.

with the other residues further from the surface, driven by the affinity of the charged termini for water.

While adsorption onto the other surfaces is weaker, marked by frequent desorption from the surface, specific residues that mediate adsorption can also be identified for these surfaces. On the Au100 surface adsorption is mediated through F20 and A21 residues. Involvement of 
only one of the phenylalanine residues may be due to the weaker adsorption of phenylalanine onto Au100 not being sufficient to overcome the unfavourable rotation around the peptide backbone for both phenylalanine residues to contact the surface. Less difference is seen for the two siilver surfaces, with the protein termini, primarily the C-terminus, being typically closer to the surface.

The different residues involved in adsorption can bee seen from considering the probability of each residue being in contact with the surface (Figure 2). These are largely higher for the Au111 surface due to the strong adsorption, with the contact probabilities for F19 and F20 being close to one. For the Au111 surface the terminal residues (K16 and E22) and the L17 also have a significant probability of contact with the surface. The contact probabilities for the other surfaces are lower and are typically larger for residues towards the C-terminus.

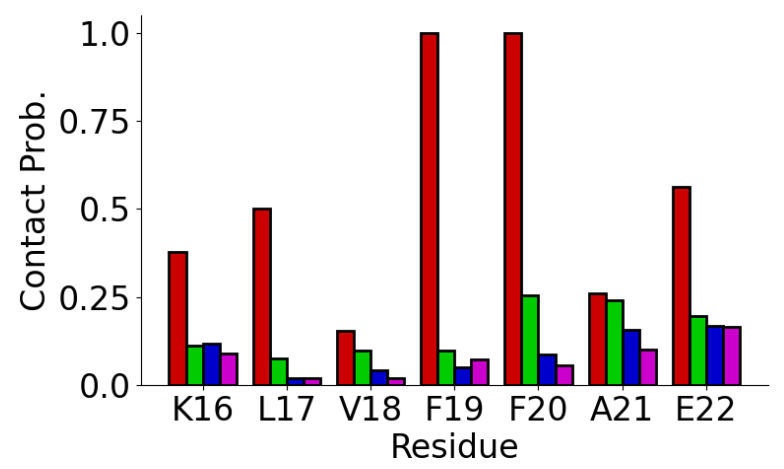

FIG. 2. Residue-surface contact probabilites for A $\beta$ (16-22) on Au111 (red), Au100 (green), Ag111 (blue), and Ag100 (magenta) surfaces.

\section{B. Conformation of $\mathbf{A} \beta(\mathbf{1 6 - 2 2})$ on metal surfaces}

The binding of specific residues to the surfaces affects the conformations $\mathrm{A} \beta(16-22)$ can adopt. Qualitatively this can be seen in representative snapshots (Figure 3). For the gold surfaces the peptide lies flatter on the surface, with two (Au111) or one (Au100) phenylalanine residue in contact with the surface, in line with prior simulations ${ }^{47}$. On silver surfaces, less of the peptide is in contact with the surface, typically consisting of the terminal residues (Figure 2). Similar to bulk solution this also allows for contacts between the two termini of the peptide. 


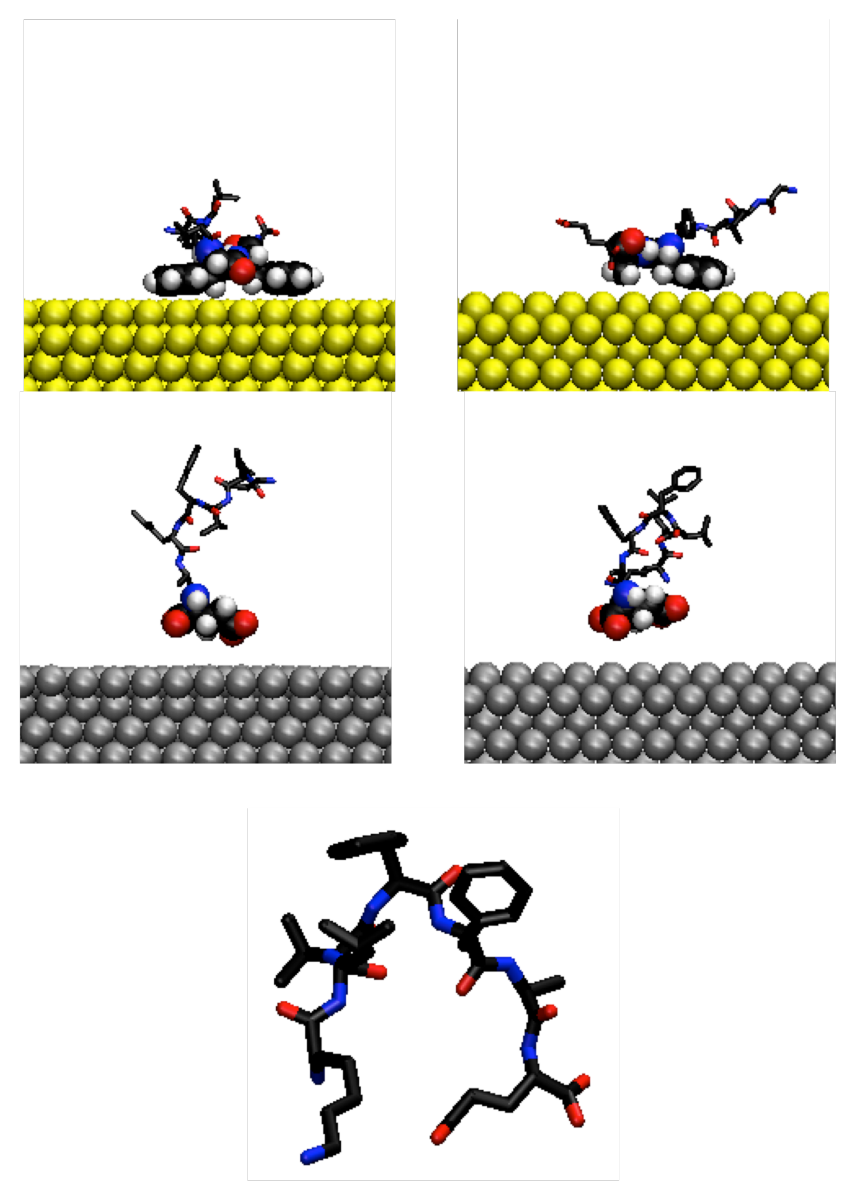

FIG. 3. Representative snapshots for $\mathrm{A} \beta(16-22)$ on Au111 (top left), Au100 (top right), Ag111(middle left), Ag100 (middle right) surfaces and in bulk solution (bottom). For the surface simulations, residues in contact with the surface are shown as VDW spheres.

The strong adsorption of the two phenylalanine residues onto the Au111 surface enhances the formation of cis-conformations compared to other surfaces and bulk solutions. This can be seen from the histogram of the angle $\phi$ between the phenylalanine side chains (Figure 4). All the systems have a peak in this near $\cos \phi=1$ but this is largest for the Au111 surface and the probability of $\cos \phi>0$ (corresponding to the cis-state) is higher for the Au111 surface. For $\cos \phi<0$, which corresponds to the trans-state the probability is lower for the Au111 compared to the other systems with $P(\cos \phi)$ going to zero at $\cos \phi=-1$. This enhancement of cis-conformers has been seen in previous studies of $\mathrm{A} \beta(16-22)$ on interfaces $^{47,80}$ and of the 
diphenylalanine dipeptide ${ }^{81}$ at the air-water interface.

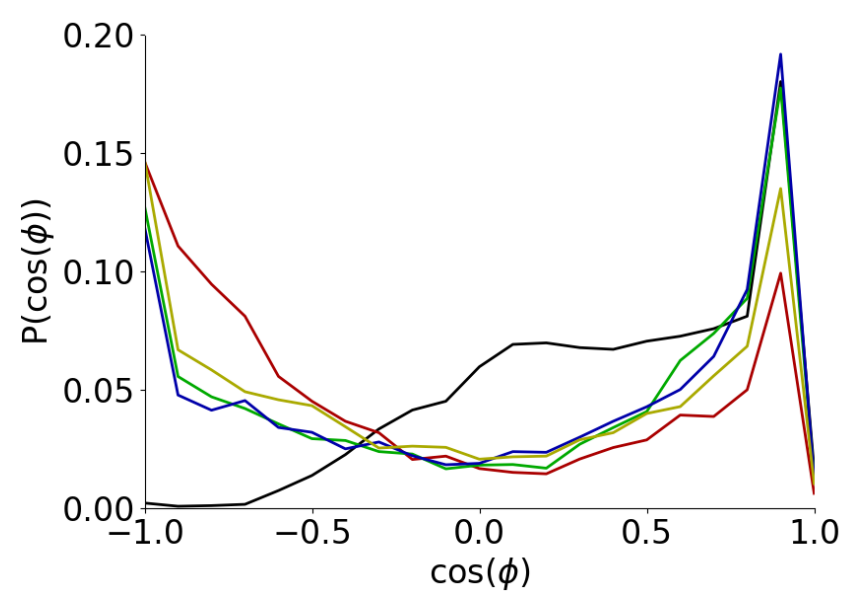

FIG. 4. Probability distribution of angle between phenylalanine side chains for Au111 (black), Au100 (red), Ag111 (green), Ag100 (blue) surfaces and bulk solution (yellow).

While the adsorption of the phenylalanine residues to the silver surfaces is lower these also have a higher probability of forming the cis-conformers. In these cases it may be driven by the tendency of the two terminal residues to adsorb to the surface (Figure 1(c)).

The preponderance of the cis-conformer leads the peptide to adopt U-shaped conformations, which brings the ends of the peptide closer together. This can be seen by the end-to-end length (Table II), which is lower for the Au111 surface than in bulk solution. By contrast $\mathrm{A} \beta(16-22)$ has a higher average end-to-end length on the Au100 surface indicating a preference for more extended conformations, which are favoured by when the phenylalanine residues adopt the trans-conformation. For the silver surfaces $R_{e e}$ is also on average lower than in bulk solution, driven by the adsorption of the terminal residues to the surface.

The less linear conformations adopted on some of the surfaces can also change the interactions between different residues in the peptide. To examine this the hydrogen bonds between different residues have been determined (Table II). For all systems hydrogen bonding between the two terminal residues (K16 and E22) is found, although only for the Au111 surface is this present in the majority of the simulation. The persistence of this is likely driven by non-linear conformations caused by the diphenylalanine motif. This is the only hydrogen bond seen for the two gold surfaces, while other hydrogen bonds are found on the other systems. 


\begin{tabular}{|c|c|c|}
\hline & $R_{e e} / \AA$ & Hydrogen bonds \\
\hline Au111 & $13.74 \pm 3.3$ & K16-E22 (0.693) \\
\hline Au100 & $15.4 \pm 4.1$ & $\mathrm{~K} 16-\mathrm{E} 22(0.211)$ \\
\hline Ag111 & $14.2 \pm 4.4$ & K16-E22 (0.231), L17-F20 (0.202) \\
\hline $\operatorname{Ag} 100$ & $13.6 \pm 4.2$ & $\begin{array}{l}\text { K16-E22 (0.296), L17-F20 (0.228), } \\
\text { V18-F20 (0.178) }\end{array}$ \\
\hline Solution & $15.2 \pm 4.1$ & K16-E22 (0.128), L17-F20 (0.109) \\
\hline
\end{tabular}

TABLE II. Average end-to-end lengths and common hydrogen bonds. Numbers in parenthesies give probabilities of each hydrogen bond.

As well as hydrogen bonding contacts between the two terminal residues are driven by attractive electrostatic interactions. Shown in Figure 5 are the histograms for the separation between the two terminal residues $\left(R_{\text {term }}\right)$, defined as being the minimum distance between either of the positively charged nitrogen atoms in K16 and any of the negatively charged oxygen atoms in E22. These have a peak at $R_{\text {term }} \sim 2.5 \AA$ corresponding to close contacts. This peak is smaller for Au100 and in bulk solution, indicating a lower probability of contacts in these cases. The second peak at $R_{\text {term }} \sim 5 \AA$ is also weaker for Au100 and solution. Notably in all cases contacts between nitrogen and oxygen atoms in the Lys and Glu side chains are more common than between the nitrogen and oxygen atoms in the charged end groups, suggesting the changes to the charge state of the termini (e.g. through neutral capping groups) is likely to be small. This is a consequence of having two charged residues at the end of the peptide; for other cases, where the side chains of the terminal residues are uncharged, changes to the charge states of the termini (e.g. through capping groups) would potentially have a larger effect ${ }^{82}$.

On the Au100 surface A $\beta(16-22)$ adopts more extended conformations with a higher probability of trans-conformers so it may be expected that it forms more fibril-like structures. To quantify this we examine the $\mathrm{C} \alpha$-C $\alpha$ distance RMSD (DRMSD) between the simulation structure and experimental fibril structure (from PDB code $2 \mathrm{y} 29^{83}$ ). This is calculated using

$$
D R M S D=\sqrt{\frac{1}{N_{C \alpha-C \alpha}} \sum_{i}\left(r_{C \alpha-C \alpha, i}^{s i m}-r_{C \alpha-C \alpha, i}^{e x p}\right)^{2}}
$$

where the sum is over all the $C \alpha-C \alpha$ pairs in the peptide. This suggests that the gold 


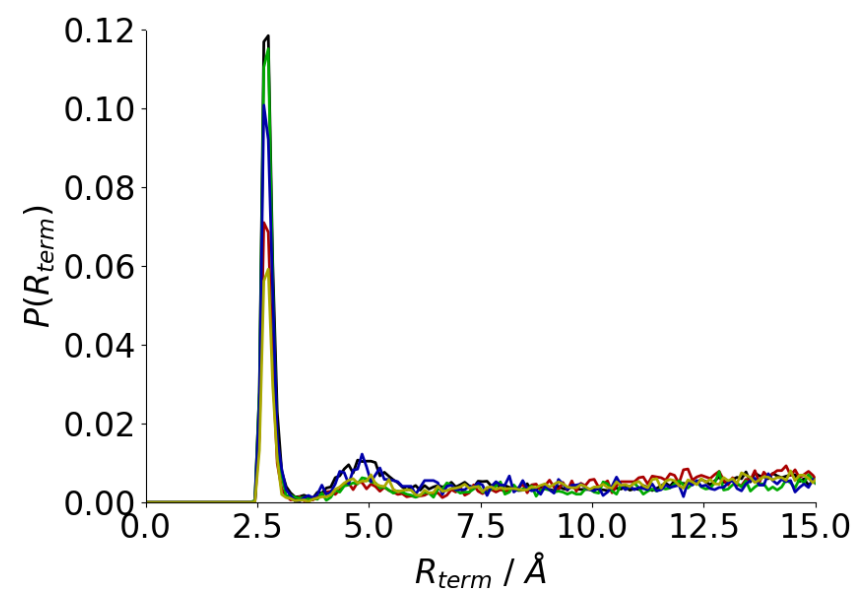

FIG. 5. Histogram of separation between termini $\left(R_{\text {term }}\right)$ for Au111 (black), Au100 (red), Ag111 (green), Ag100 (blue) surfaces and bulk solution (yellow).

surfaces have a differing effect on the formation of fibril-like structures (Figure 6), with fibril-like structures being more common on the Au100 surface. By contrast fibril-like conformations are unlikely on the Au111 surface. The inhibition of fibril-like conformations on the Au111 surface is consistent with previous simulation work ${ }^{47}$. While both gold surfaces differ substantially from bulk solution for the two silver surfaces there is less difference to solution. The formation of fibril-like structures is also similar for the Ag111 and Ag100 surfaces, suggesting a smaller facet dependence compared to gold.

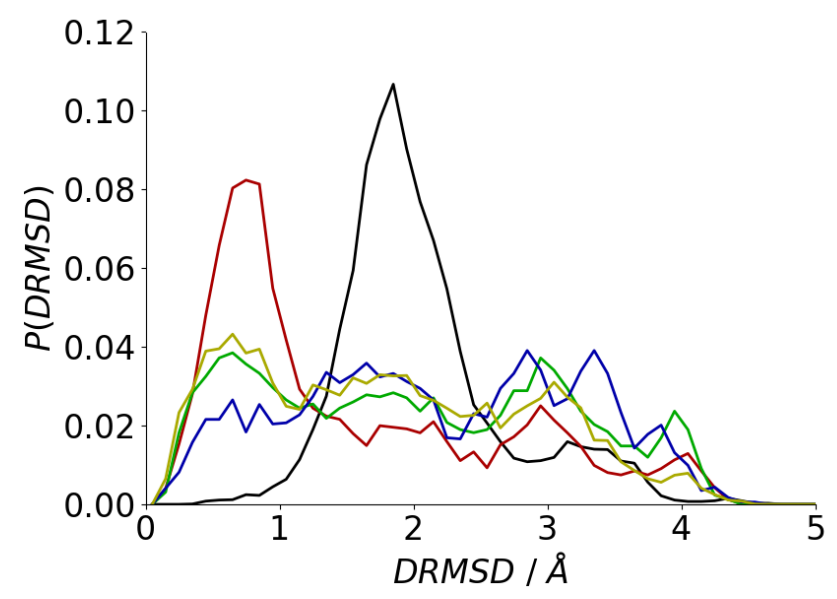

FIG. 6. Histogram of DRMSD relative to experimental fibril structure for Au111 (black), Au100 (red), Ag111 (green), Ag100 (blue) surfaces and bulk solution (yellow). 


\section{Adsorption onto metal surfaces affects the conformational ensemble of $\mathbf{A} \beta$ (16-}

22)

As an intrinsically disordered peptide $\mathrm{A} \beta(16-22)$ forms an ensemble of different conformations. Both the set of conformations and the size of these can be affected by surfaces. For the different systems the conformational ensemble has been determined using a cluster analysis. Shown in Table III are the number of distinct clusters found for the different systems. Notably $N_{\text {clusters }}$ is smaller for all surfaces compared to bulk solution, due to the binding of specific residues to the surfaces (Figure 1(c)) which limits the possible conformations, which has been seen in previous simulations of intrinsically disordered proteins on surfaces ${ }^{50,80}$. This is particularly noticable for the Au111 surface due to the strong binding of the two Phe residues to the surface. The number of conformations is higher for the other surfaces, which may be reflective of the weaker binding of $\mathrm{A} \beta(16-22)$ to these surfaces. While the cluster analysis is restricted to the conformations where the peptide is adsorbed to the surface, the ability of the peptide to desorb from the surface may also give $\mathrm{A} \beta(16$-22) greater conformational freedom on these surfaces compared to the Au111 surface. Again a smaller difference is seen between the two silver surfaces.

\begin{tabular}{ccc}
\hline & $N_{\text {clusters }}$ & $S_{\text {conf }} / k_{\mathrm{B}}$ \\
\hline Au111 & 93 & 2.54 \\
Au100 & 157 & 3.54 \\
Ag111 & 156 & 3.72 \\
Ag100 & 163 & 3.84 \\
Solution & 260 & 4.18 \\
\hline
\end{tabular}

TABLE III. Number of clusters and conformational entropy determined from cluster analysis over last 100 ns of simulations.

Changes in the conformational ensemble can also be seen in the conformational entropy (Table III). This is lower for all the surfaces compared to bulk solution and is considerably lower for Au111 compared to the other surfaces. The higher conformational entropy on silver surfaces compared to gold and the higher values on 100 compared to 111 surfaces is consistent with prior simulatons of gold and silver binding peptides ${ }^{57}$. 
The probability of each cluster in the simulations can be used to determine the free energy of each cluster

$$
\Delta F=-k_{\mathrm{B}} T \ln \frac{P_{i}}{P_{1}}
$$

where $\Delta F$ is the free energy difference between the $i$ th and 1 st cluster and $P_{i}$ is the probability of the $i$ th cluster. For the silver surfaces and bulk solution the free energy varies similarly with cluster ID, showing a relatively slow increase consistent with a number of conformations with similar free energies. On the gold surfaces there are fewer low energy states, suggesting that there is a greater restriction on the different conformations.

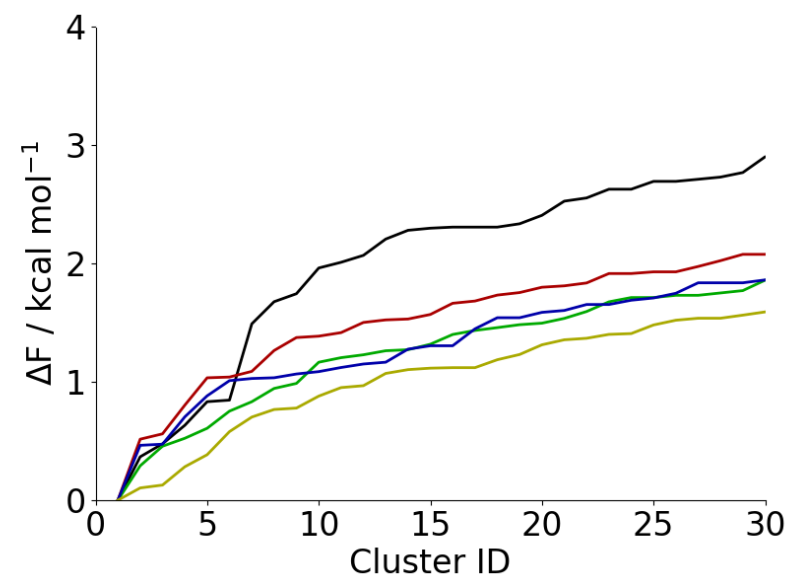

FIG. 7. Free energy of clusters for Au111 (black), Au100 (red), Ag111 (green), Ag100 (blue), and solution (yellow).

The cumulative probability of different conformations has been used to classify peptides in terms of their binding entropy ${ }^{84}$. For the Au100, Ag111, and Ag100 surfaces the cumulative probability of the top five clusters is under $60 \%$ classifying $\mathrm{A} \beta(16-22)$ as a high entropy binder on these surfaces. By the same scheme $\mathrm{A} \beta(16-22)$ is a medium entropy binder on the Au111 (the cumulative probability of the top five clusters is over $60 \%$ but for the top three clusters it is under $75 \%$ ). In combination with the difference with the interaction energies between the surfaces, this suggests a qualitative difference in the behaviour of $\mathrm{A} \beta(16-22)$ on the Au111 surface.

To examine how similar the conformational ensembles are for the different systems we perform a cluster analysis on the combination of the different systems. Comparing the probability of the different conformations in each simulation (Figure 8(a)) shows that the 
conformational ensembles on the two gold surfaces are significantly different to the other systems. Notably on the Au111 surface there are fewer conformations that are significantly populated, consistent with the lower conformational entropy. The most populated conformations for the Au111 and Au100 surfaces are also typically less likely on the silver surfaces and in bulk solution.

(a)

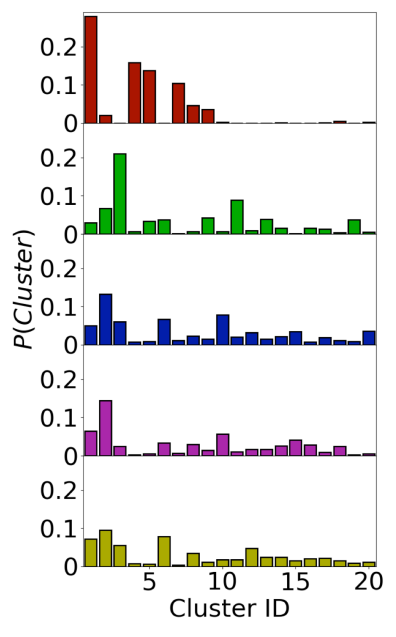

(b)
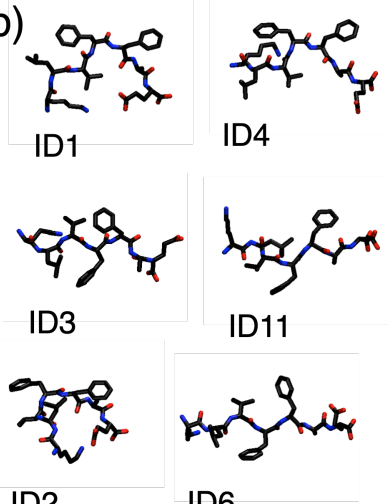

ID2

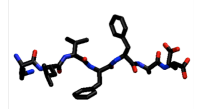

ID6

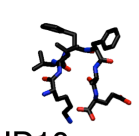

ID10

FIG. 8. (a) Probability of highest ranked clusters for (top to bottom) Au111, Au100, Ag111, Ag100, and bulk solution. (b) Snapshots of selected conformations found from the combined cluster analysis.

The observation that $P($ Cluster $)$ for the two gold surfaces differ from each other and the other surfaces suggests that we can characterise the adsorption of $\mathrm{A} \beta(16-22)$ onto gold as an example of induced fit-like behaviour ${ }^{85}$, where the surface causes the peptide to adopt different conformations. In the case of $\mathrm{A} \beta(16-22)$ on gold this set of conformations also depends on the particular exposed facet. The close similarity between the distributions on the silver surfaces and bulk solution suggests that the adsorption of A $\beta(16-22)$ onto silver is characterised by conformational selection ${ }^{86}$, where similar conformations are found with, in this case minor, changes in likelihood of the different conformations.

The structures commonly found for the different systems (Figure 8(b)) can be related to the typical interaction between the peptide and surface. For the Au111 surface the most common conformations (ID 1,4) have the two phenylalanine residues in the cis-conformation, 
with the two rings in the same plane, which maximise their ability to contact the gold surface. The most common conformations for the Au100 surface (e.g. 3, 11) include the more extended, fibril-like structures. On the silver surfaces and in bulk solution, both extended and more compact conformations are found. Many of the conformations found on the silver surfaces and in bulk solution typically have contacts between the terminal residues, driven by electrostatic attraction between these.

\section{CONCLUSIONS}

Using replica exchange molecular dynamics simulations we have investigated the conformation of the model amyloidogenic peptide, $\mathrm{A} \beta(16-22)$ on gold and silver surfaces. We found significant differences in behaviour between the Au111 and Au100 surfaces, with the peptide adsorption significantly stronger on the Au111 surface. This is due to the strong interaction between phenylalanine residues and the Au111 surface, with the weaker interaction between these and the Au100 surface leading to weaker adsorption. By contrast a smaller difference between the two silver surfaces (Ag111 and Ag100) was seen, suggesting that adsorption onto silver is less dependent on the exposed facet. The contrasting facet dependence of adsorption is similar to that seen for gold and silver binding peptides.

Comparison of the peptide-surface interaction energies and conformational entropy suggest that despite the structural similarities between gold and silver surfaces the binding of A $\beta$ (16-22) to these have different driving forces. For gold surfaces $E_{\text {int }}$ and $S_{\text {conf }}$ suggest that this is largely enthalpically driven, while on silver surfaces it is driven by entropy. This is in line with previous simulation studies of gold and silver binding peptides ${ }^{57}$. The difference between these is smaller for the 100 facets compared to the 111 facets.

Adsorption onto surfaces affects the conformations adopted by $\mathrm{A} \beta(16-22)$. As an intrinsitically disordered peptide it adopts a range of different conformations (conformational ensemble). The size of this ensemble is smaller on surfaces compared to bulk solution, as the surface restricts the possible conformations of the peptide. The Au111 surface has the smallest number of conformations, as the strong adsorption of the phenylalanine residues effectively pins these to the surface. Comparison of the conformational ensembles for the different surfaces and bulk solution shows that the gold surfaces induce different sets of conformations to the silver surfaces and bulk solution. This suggests that we can describe 
adsorption onto gold surfaces as induced fit-like while adsorption onto silver surfaces can be thought of as conformational selection. It may be argued that induced fit-like behaviour is more likely for cases with strong adsorption, consistent with previous simulations of IDPs on hydrophibic and hydrophilic self-assembled monolayers ${ }^{50}$.

The conformations of $\mathrm{A} \beta(16-22)$ adopted on different surfaces has implications for how they affect peptide fibrilliation. On the Au111 surface A $\beta(16-22)$ adopts structures that are quite different to those of $\mathrm{A} \beta(16-22)$ in amyloid fibrils, while it is more likely to adopt fibrillike conformations on the Au100 surface. This suggests that even for the same material, different surface faces may have differing effects on fibrillation. As the proportion of 111 and 100 faces on nanoparticles varies with their size ${ }^{87}$, this may explain the size dependent effect of gold nanoparticles on the formation of amyloid fibrils ${ }^{24}$. The smaller difference between the two silver surfaces suggests that nanoparticle size may have a smaller effect for silver compared to gold.

While the results of this are consistent with previous simulation results, the behaviour of peptides and proteins on surfaces depends on both the surface properties and on the peptide

sequence. It would also be interesting to extend this to the examination of other metal surfaces to consider how changes to these structurally similar systems affect the behaviour of amyloidogenic peptides.

\section{ACKNOWLEDGEMENTS}

Gromacs files containing the AgP-charmm force field were kindly provided by Prof. Tiffany Walsh (Deakin University). Computational facilities for this work were provided by the SFI/HEA funded Irish Centre for High End Computing (project code ngche092b). KS was supported by the Irish Research Council (GOPID/2019/372).

* david.cheung@nuigalway.ie

1 M. G. Iadanza, M. P. Jackson, E. W. Hewitt, N. A. Ranson, and S. E. Radford, Nat Rev Mol Cell Biol 19, 755 (2018).

2 P. C. Ke, R. Zhou, L. C. Serpell, R. Riek, T. P. J. Knowles, H. A. Lashuel, E. Gazit, I. W. Hamley, T. P. Davis, M. Fandrich, et al., Chem. Soc. Rev. 49, 5473 (2020). 
3 P. Maresova, H. Mohelska, J. Dolejs, and K. Kuca, Current Alzheimer Research 12, 903 (2015).

4 C. Zhong, T. Gurry, A. A. Cheng, J. Downey, Z. Deng, C. M. Stultz, and T. K. Lu, Nature Nanotech 9, 858 (2014).

5 E. E. Erskine, C. E. MacPhee, and N. R. Stanley-Wall, Journal of Molecular Biology 430, 3642 (2018).

6 T. P. J. Knowles and R. Mezzenga, Advanced Materials 28, 6546 (2016).

7 C. Li, R. Qin, R. Liu, S. Miao, and P. Yang, Biomater. Sci. 6, 462 (2018).

8 K. A. Burke, E. A. Yates, and J. Legleiter, Front. Neurol. 4, 17 (2013).

9 A. Keller and G. Grundmeier, Applied Surface Science 506, 144991 (2020).

10 M. Stefani, Neuroscientist 13, 519 (2007).

11 E. Terzi, G. Holzemann, and J. Seelig, Biochemistry 36, 14845 (1997).

12 V. Koppaka and P. H. Axelsen, Biochemistry 39, 10011 (2000).

13 M. Bokvist, F. Lindstrom, A. Watts, and G. Grabner, Journal of Molecular Biology 335, 1039 (2004).

14 Y. Verdier, M. Zarandi, and B. Penke, Journal of Peptide Science 10, 229 (2004).

15 S. A. Jayasinghe and R. Langen, Biochemistry 44, 12113 (2005).

16 L. Jean, C. F. Lee, C. Lee, M. Shaw, and D. J. Vaux, The FASEB Journal 24, 309 (2010).

17 S. Bolisetty and R. Mezzenga, Nature Nanotech 11, 365 (2016).

18 C. A. E. Hauser, S. Maurer-Stroh, and I. C. Martins, Chem. Soc. Rev. 43, 5326 (2014).

19 Q. Zhang, S. Bolisetty, Y. Cao, S. Handschin, J. Adamcik, Q. Peng, and R. Mezzenga, Angewandte Chemie International Edition 58, 6012 (2019).

20 T. Cedervall, I. Lynch, S. Lindman, T. Berggard, E. Thulin, H. Nilsson, K. a. Dawson, and S. Linse, Proceedings of the National Academy of Sciences 104, 2050 (2007).

21 C. Cabaleiro-Lago, F. Quinlan-Pluck, I. Lynch, K. A. Dawson, and S. Linse, ACS Chemical Neuroscience 1, 279 (2010).

22 A. Gladytz, M. Wagner, T. Haupl, C. Elsner, and B. Abel, Part. Part. Syst. Charact. 32, 573 (2015).

23 A. Gladytz, B. Abel, and H. J. Risselada, Angew. Chem. Int. Ed. 55, 11242 (2016).

24 G. Gao, M. Zhang, D. Gong, R. Chen, X. Hu, and T. Sun, Nanoscale 9, 4107 (2017).

25 T. John, A. Gladytz, C. Kubeil, L. L. Martin, H. J. Risselada, and B. Abel, Nanoscale 10, 20894 (2018). 
R. R. Arvizo, S. Bhattacharyya, R. A. Kudgus, K. Giri, R. Bhattacharya, and P. Mukherjee, Chemical Society Reviews 41, 2943 (2012).

27 Y. He, Z. Du, S. Ma, Y. Liu, D. Li, H. Huang, S. Jiang, S. Cheng, W. Wu, K. Zhang, et al., Int J Nanomedicine 11, 1879 (2016).

28 Q. Cheng and Y. Liu, WIREs Nanomedicine and Nanobiotechnology 9, e1410 (2017).

29 D. Pedone, M. Moglianetti, E. D. Luca, G. Bardi, and P. Paolo-Pompa, Chemical Society Reviews 46, 4951 (2017).

30 P. Puja and P. Kumar, Spectrochimica Acta Part A: Molecular and Biomolecular Spectroscopy 211, 94 (2019).

31 S. K. Sonawane, A. Ahmad, and S. Chinnathambi, ACS Omega 4, 12833 (2019).

32 R. Coppage, J. M. Slocik, B. D. Briggs, A. I. Frenkel, H. Heinz, R. R. Naik, and M. R. Knecht, J. Am. Chem. Soc. 133, 12346 (2011).

33 Z. E. Hughes, R. Kochandra, and T. R. Walsh, Langmuir 33, 3742 (2017).

34 J. Schneider and L. Colombi Ciacchi, Journal of the American Chemical Society 134, 2407 (2012).

35 L. Dong, Q. Luo, K. Cheng, H. Shi, Q. Wang, W. Weng, and W.-Q. Han, Sci Rep 4, 5084 (2014).

36 Y. Li and Y. Huang, Advanced Materials 22, 1921 (2010).

37 C.-Y. Chiu, Y. Li, L. Ruan, X. Ye, C. B. Murray, and Y. Huang, Nature Chemistry 3, 393 (2011).

38 S.-T. Wang, Y. Lin, N. Todorova, Y. Xu, M. Mazo, S. Rana, V. Leonardo, N. Amdursky, C. D. Spicer, B. D. Alexander, et al., Chemistry of Materials 29, 1550 (2017).

39 L. Shen, T. Adachi, D. Vanden Bout, and X.-Y. Zhu, J. Am. Chem. Soc. 134, 14172 (2012).

40 A. Keller, M. Fritzsche, Y. P. Yu, Q. Liu, Y. M. Li, M. Dong, and F. Besenbacher, ACS Nano 5, $2770(2011)$.

41 R. Hajiraissi, I. Giner, G. Grundmeier, and A. Keller, Langmuir 33, 372 (2016).

42 R. Hajiraissi, M. Hanke, Y. Yang, B. Duderija, A. Gonzalez Orive, G. Grundmeier, and A. Keller, Langmuir 34, 3517 (2018).

43 T. Ando, Current Opinion in Structural Biology 28, 63 (2014).

44 S. Hosseinpour, S. J. Roeters, M. Bonn, W. Peukert, S. Woutersen, and T. Weidner, Chem. Rev. 120, 3420 (2020). 
45 M. Ozboyaci, D. B. Kokh, S. Corni, and R. C. Wade, Quarterly Reviews of Biophysics 49, 1 (2016).

46 T. R. Walsh, Accounts of Chemical Research 50, 1617 (2017).

47 L. Bellucci, A. Ardèvol, M. Parrinello, H. Lutz, H. Lu, T. Weidner, and S. Corni, Nanoscale 8, 8737 (2016).

48 L. Bellucci, G. Bussi, R. Di Felice, and S. Corni, Nanoscale 9, 2279 (2017).

49 F. Tavanti, A. Pedone, and M. C. Menziani, IJMS 22, 26 (2020).

50 D. L. Cheung, Biointerphases 15, 051001 (2020).

51 G. Brancolini, A. Corazza, M. Vuano, F. Fogolari, M. C. Mimmi, V. Bellotti, M. Stoppini, S. Corni, and G. Esposito, ACS Nano 9, 2600 (2015).

52 D. J. Earl and M. W. Deem, Physical Chemistry Chemical Physics 7, 3910 (2005).

53 A. Laio and F. L. Gervasio, Reports on Progress in Physics 71, 126601 (2008).

54 H. Heinz, K. C. Jha, J. Luettmer-Strathmann, B. L. Farmer, and R. R. Naik, J. R. Soc. Interface. 8, 220 (2011).

55 F. Iori and S. Corni, J. Comput. Chem. 29, 1656 (2008).

56 S. Corni, J. Comput. Chem. 38, 2130 (2017).

57 J. P. Palafox-Hernandez, Z. Tang, Z. E. Hughes, Y. Li, M. T. Swihart, P. N. Prasad, T. R. Walsh, and M. R. Knecht, Chem. Mater. 26, 4960 (2014).

58 L. B. Wright, J. P. Palafox-Hernandez, P. M. Rodger, S. Corni, and T. R. Walsh, Chem. Sci. 6, $5204(2015)$.

59 J. J. Balbach, Y. Ishii, O. N. Antzutkin, R. D. Leapman, N. W. Rizzo, F. Dyda, J. Reed, and R. Tycko, Biochemistry 39, 13748 (2000).

60 O. A. Perfilieva, D. V. Pyshnyi, and A. A. Lomzov, J. Chem. Theory Comput. 15, 1278 (2019).

61 L. B. Wright, P. M. Rodger, T. R. Walsh, and S. Corni, Journal of Physical Chemistry C 117, $24292(2013$.

62 Z. E. Hughes, L. B. Wright, and T. R. Walsh, Langmuir 29, 13217 (2013).

63 A. D. MacKerell, D. Bashford, R. L. Dunbrack, J. D. Evanseck, M. J. Field, S. Fischer, J. Gao, H. Guo, S. Ha, D. Joseph-McCarthy, et al., The Journal of Physical Chemistry B 102, 3586 (1998).

64 P. Bjelkmar, P. Larsson, M. A. Cuendet, B. Hess, and E. Lindahl, J. Chem. Theory Comput. 6, 459 (2010). 
S. Piana, K. Lindorff-Larsen, and D. Shaw, Biophysical Journal 100, L47 (2011).

66 W. L. Jorgensen, J. Chandrasekhar, and J. D. Madura, J. Chem. Phys. 79, 926 (1983).

67 V. H. Man, X. He, P. Derreumaux, B. Ji, X.-Q. Xie, P. H. Nguyen, and J. Wang, J. Chem. Theory Comput. 15, 1440 (2019).

68 L. B. Wright, P. M. Rodger, S. Corni, and T. R. Walsh, Journal of Chemical Theory and Computation 9, 1616 (2013.

69 P. Liu, B. Kim, R. A. Friesner, and B. J. Berne, Proceedings of the National Academy of Sciences of the United States of America 102, 13749 (2005).

70 L. Wang, R. A. Friesner, and B. J. Berne, Journal of Physical Chemistry B 115, 9431 (2011.

71 Y. Sugita and Y. Okamoto, Chemical Physics Letters 314, 141 (1999).

72 G. Bussi, D. Donadio, and M. Parrinello, Journal of Chemical Physics 126, 014101/1 (2007).

73 M. Parrinello and A. Rahman, Journal of Applied Physics 52, 7182 (1981).

74 M. J. Abraham, T. Murtola, R. Schulz, S. Páll, J. C. Smith, B. Hess, and E. Lindahl, SoftwareX 1-2, $19(2015)$.

75 G. A. Tribello, M. Bonomi, D. Branduardi, C. Camilloni, and G. Bussi, Computer Physics Communications 185, 604 (2014).

76 G. Bussi, Molecular Physics 112, 379 (2013).

77 X. Daura, K. Gademann, B. Jaun, D. Seebach, W. F. Van Gunsteren, and A. E. Mark, Angew. Chemie Int. Ed. 38, 236 (1999).

78 N. Michaud-Agrawal, E. J. Denning, T. B. Woolf, and O. Beckstein, Journal of computational chemistry 32, 2319 (2011).

79 W. Humphrey, A. Dalke, and K. Schulten, Journal of Molecular Graphics 14, 33 (1996).

80 S. Samantray and D. L. Cheung, Biointerphases 15, 061011 (2020).

81 C. Dalgicdir, O. Sensoy, C. Peter, and M. Sayar, The Journal of Chemical Physics 139, 234115 (2013).

82 R. Hajiraissi, M. Hanke, A. Gonzalez Orive, B. Duderija, U. Hofmann, Y. Zhang, G. Grundmeier, and A. Keller, ACS Omega 4, 2649 (2019).

83 J.-P. Colletier, A. Laganowsky, M. Landau, M. Zhao, A. B. Soriaga, L. Goldschmidt, D. Flot, D. Cascio, M. R. Sawaya, and D. Eisenberg, Proceedings of the National Academy of Sciences 108, 16938 (2011). 
84 Z. Tang, J. P. Palafox-Hernandez, W.-C. Law, Z. E. Hughes, M. T. Swihart, P. N. Prasad, M. R. Knecht, and T. R. Walsh, ACS Nano 7, 9632 (2013).

85 D. E. Koshland, Proc. Natl. Acad. Sci. 44, 98 (1958).

86 S. Kumar, B. Ma, C.-J. Tsai, N. Sinha, and R. Nussinov, Protein Science 9, 10 (2008).

87 A. S. Barnard and Y. Chen, J. Mater. Chem. 21, 12239 (2011). 